\title{
THE REFRACTIVE INDEX ALONG THE OPTIC AXIS OF THE BOVINE LENS
}

\author{
B. K. PIERSCIONEK \\ Bundoora, Australia
}

\section{SUMMARY}

This study aimed to measure the refractive index values along the optic axes of adult bovine lenses. An optic fibre reflectometric sensor was used for this purpose. Seventeen lenses were examined and over the weight range studied there were no apparent age-related trends in the index gradient. The peak index values varied from 1.432 to 1.442 . There was a greater variation in the edge index values, which may have reflected differences in hydration on lens surfaces or may be indicative of a slight decrease in surface index with age.

In the field of visual optics there remains a paucity of knowledge about the optical properties of the lens. A major factor which has limited our understanding of lenticular optics, and hence of age-related impairments such as opacification of the lens (cataract) and the gradual depletion of near focussing ability (presbyopia), is the difficulty in determining the lenticular refractive index. This arises because the index is not uniform but increases from the periphery of the lens to the centre.

Previous methods of measurement have been either (a) destructive requiring tissue slicing or bisection; ${ }^{1-7}$ (b) based on computer simulations; ${ }^{8}$ or (c) involved with tracing light rays through lenses and calculating the index gradient from ray path parameters. $^{9-13}$ The technique employed to determine the gradient accurately needs to minimise tissue violation in order to avoid any alteration to the hydration of the tissue and hence to the index gradient. Although computer simulations and mathematical ray trace analyses are non-destructive, they contain inherent assumptions and approximations which may introduce inaccuracies in the derived

From: Department of Electronic Engineering, Latrobe University, Bundoora, and National Vision Research Institute, Carlton, Victoria, Australia.

Correspondence to: B. K. Pierscionek, Department of Electronic Engineering, Latrobe University, Bundoora, Victoria 3083, Australia. results. With ray tracing, in some species, added assumptions are needed when calculating the refractive index gradient in the sagittal plane because in this plane the lens shape is asymmetric.

What is required is a method which can directly measure the refractive index, with a minimum degree of tissue violation, at any point on the surface of and within the lens. Recently a reflectometric fibre optic sensor has been developed which permits very localised measurements of refractive index to be made. ${ }^{14}$ It has been used to determine the refractive index on the surfaces of human ${ }^{15}$ and decapsulated bovine lenses. ${ }^{16}$ The sensing technique has now been extended to enable determination of the index variations within the lens: the index gradient. This paper reports the results of refractive index measurement along the optic axes of adult bovine lenses.

\section{METHODS}

The bovine lenses were obtained from abattoirs and all used within 8 hours of death. Seventeen lenses were investigated, ranging in pre-experimental weight from $1.676 \mathrm{~g}$ to $2.646 \mathrm{~g}$. Lenses were weighed immediately after removal from the eye.

Sagittal widths (the thickness of the lens from the anterior to the posterior pole) were measured with a millimetre scale and lenses were set in an $0.5 \%$ agarose gel in a physiological buffer ${ }^{11}$ with the equatorial plane horizontal, leaving either the anterior or posterior surface exposed for measurement. In 16 lenses the gradient was measured along both anterior and posterior parts of the optic axis; in 1 lens the anterior section only was measured. The top surfaces of the sides of the cell in which the lenses were set were demarcated in $0.5 \mathrm{~mm}$ steps. This assisted in positioning of the optic fibre probe (described later) above the centre of the lens. When the probe was set above the pole from which the measurements were to begin, the ray path through the lens did not deviate. Equatorial diameters were determined by measuring the 
distance moved by the probe as the light reflex traversed from one end of the lens to the other.

\section{Apparatus and Technique}

The instrumentation has been described previously ${ }^{14-16}$ but will be briefly explained here. It comprises a $670 \mathrm{~nm}, 1 \mathrm{~mW}$ diode laser (Glotracr (GT670FC), Fibrenet, Rowville, Victoria, Australia) to which is attached one arm of a $2 \times 2$ optic fibre coupler (50:50 for $670 \mathrm{~nm}$ with single mode Flexcor fibre arms: $125 \mu \mathrm{m}$ in diameter with a core diameter of $4 \mu \mathrm{m}$; Amphenol, Lisle, IL). A coupler consists of (in this case) two optical fibres joined over a small section to enable light transfer from one fibre to another. The function of the coupler is to split the light signal and it is therefore analogous to a beam splitter. The coupler serves the function of allowing reflected light initially to travel back along the same path as the incident light and at the region of coupling to be deflected to a detector. The end of the fibre which meets the sample is flat for optimum reflection and encased in a 26 gauge needle (Terumo syringe needle) with a polished, flattened end. This is referred to as the experimental probe. The probe is attached to a three-way translation stage with manual micrometer adjustment.

Light reflected from the probe/sample interface returns through the coupler and part of this is transmitted to a detector (Anritsu MA9412A, Alcatel Australia, South Melbourne, Victoria, Australia) which sends signals to a power meter (Anritsu MA9001A, Alcatel Australia). Readings of the reflected light are collected on a Macintosh computer using the data acquisition program SuperScope (GW Instruments, 35 Medford St., Somerville, MA 02143). Each measurement provided was the average of 40 readings collected, within seconds, by the data acquisition program. Two measurements were taken for every point within each lens. In most cases the readings were within $0.5 \mathrm{dBm}$ of each other. All results presented are the averages of the two measurements.

Where fluctuations were greater, up to five readings were taken until stability was reached. This was found to occur with occasional surface readings, possibly indicating changes associated with evaporation of small amounts of fluid. (Most of the fluid found seeping out from under the capsule was blotted away.)

The power meter records the reflectance, which is the ratio of the reflected light intensity to the incident light intensity. According to Fresnel's equations, for normal incidence:

$$
R=\left[\left(n_{1}-n_{2}\right) /\left(n_{1}+n_{2}\right)\right]^{2}
$$

where $R$ is the reflectance, $n_{1}$ is the refractive index of the first medium (fibre) and $n_{2}$ is the refractive index of the second medium (lens).

Any attenuations of the light signal or spurious contributions from scattering components in the fibre optic system were corrected using the equations described previously ${ }^{14}$ and provided in the Appendix.

Immediately preceding measurement, the capsule was pierced very gently at the pole (anterior or posterior depending on which side of the lens was being probed) using a 22 gauge needle (Terumo syringe needle). The anterior capsule was always more difficult to pierce than the posterior capsule. Piercing of the capsule did not cause any outflow of lens tissue. In some cases a little fluid seeped out immediately after piercing. This was gently blotted away before measurements were taken.

For every lens, before measurements on the tissue were made, a reference reading was taken in air and then the probe was moved until it touched the surface of the lens and another reading was taken. Subsequent movements through the lens tissue were made in $1 \mathrm{~mm}$ steps, with readings taken at each step, until the approximate position of the equator was reached. This was estimated from previously measured anterior and posterior sagittal width values. ${ }^{17}$ The probe was then removed from the lens, the lens turned over and measurements repeated on the other side. The order of which section, anterior or posterior, was probed first was alternated in consecutive samples.

Lenses were reweighed after completion of the experiment; the difference in weights before and after experimentation was $2 \%$ (of the pre-experimental weight) and in most cases less than $1 \%$. In one lens the weight varied by $3.5 \%$.

\section{Errors}

The maximum acceptable difference between measurements of reflected light was $0.5 \mathrm{dBm}$. The resultant error in refractive index varied depending on the index magnitude. The maximum error in refractive index was calculated to be $\pm 0.005(\max )$ and the error in translation was $\pm 0.001 \mathrm{~mm}$. The stability of the sensor over time depends on the stability of the laser diode, which varied by $0.1 \mathrm{dBm}$ over half an hour. Since the time of measurement for each section was less than 10 minutes, the variability in the light output of the diode was not detectable over the experimental time period.

\section{RESULTS}

The refractive index values at points along the optic axes in 17 anterior sections and 16 posterior sections from adult bovine lenses were measured using a reflectometric optic fibre sensor. The refractive index increases from the surface to the centre of the lens. 

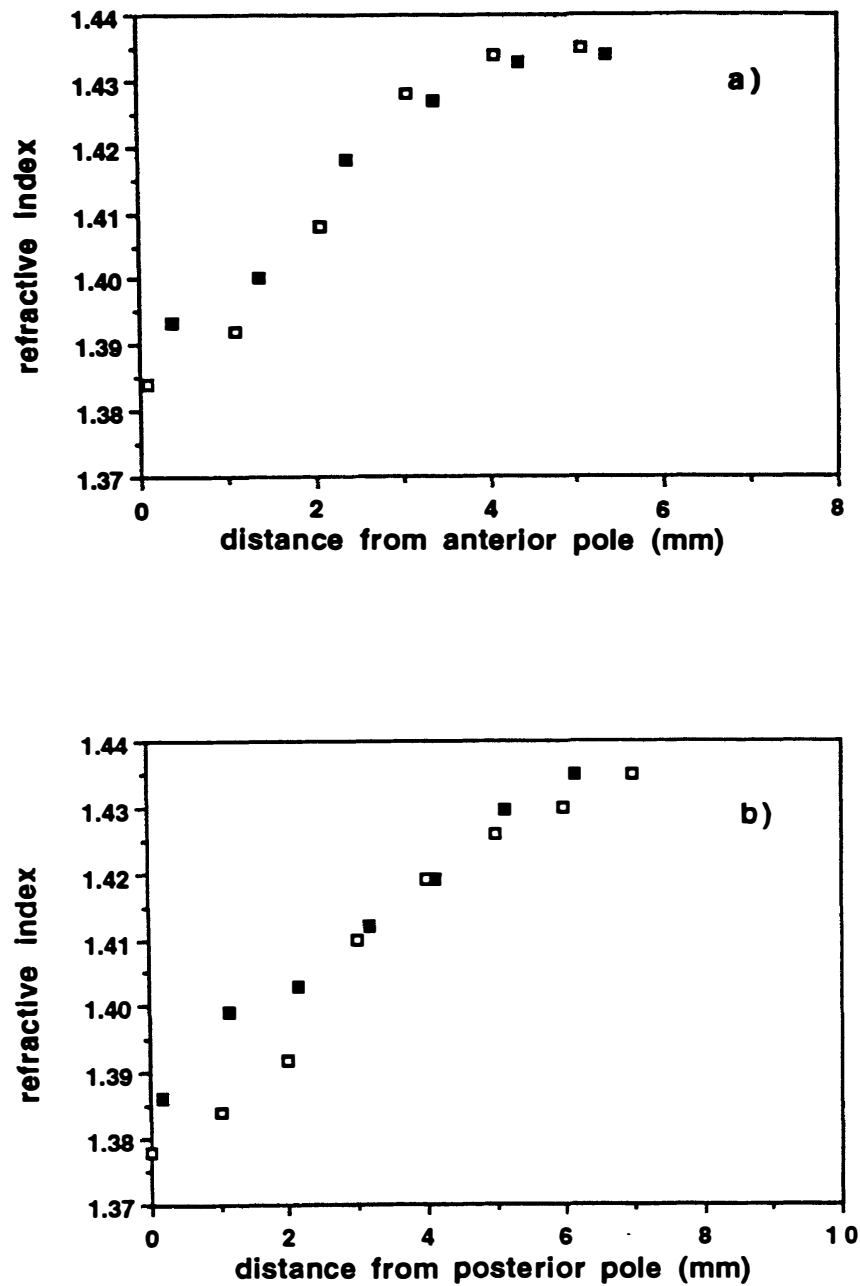

Fig. 1. Refractive index plotted against distance from (a) anterior and (b) posterior poles of two bovine lenses weighing $1.705 \mathrm{~g}$ (filled squares) and $2.605 \mathrm{~g}$ (open squares).

Index gradients from both sections of a $1.705 \mathrm{~g}$ and a $2.605 \mathrm{~g}$ lens are shown in Fig. 1a and b plotted against the distances from the poles. The gradients in

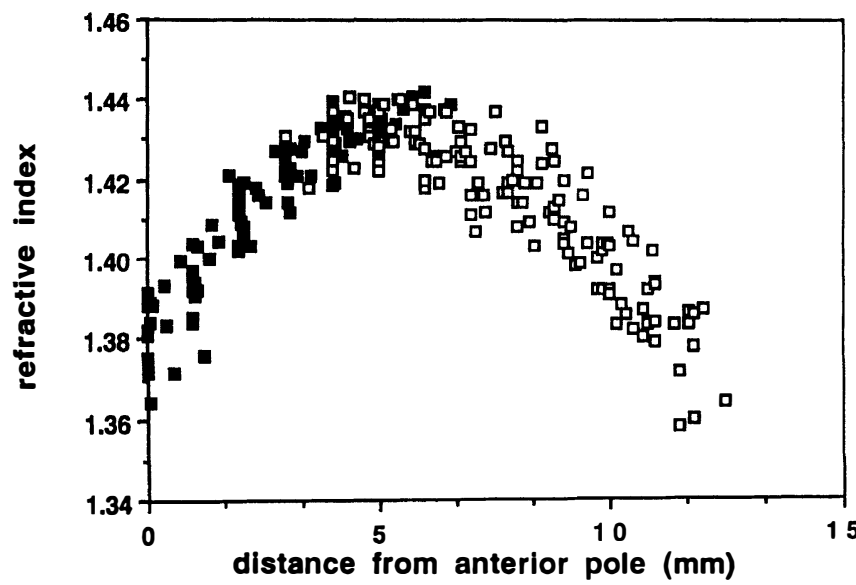

Fig. 3. Refractive index variations along the optic axis from all lenses (17 anterior, 16 posterior gradients; lens weight range 1.676-2.646 g) plotted against the distance from the anterior pole. Results from measurements made in the anterior section are designated by filled squares, those from measurements in the posterior section by open squares.
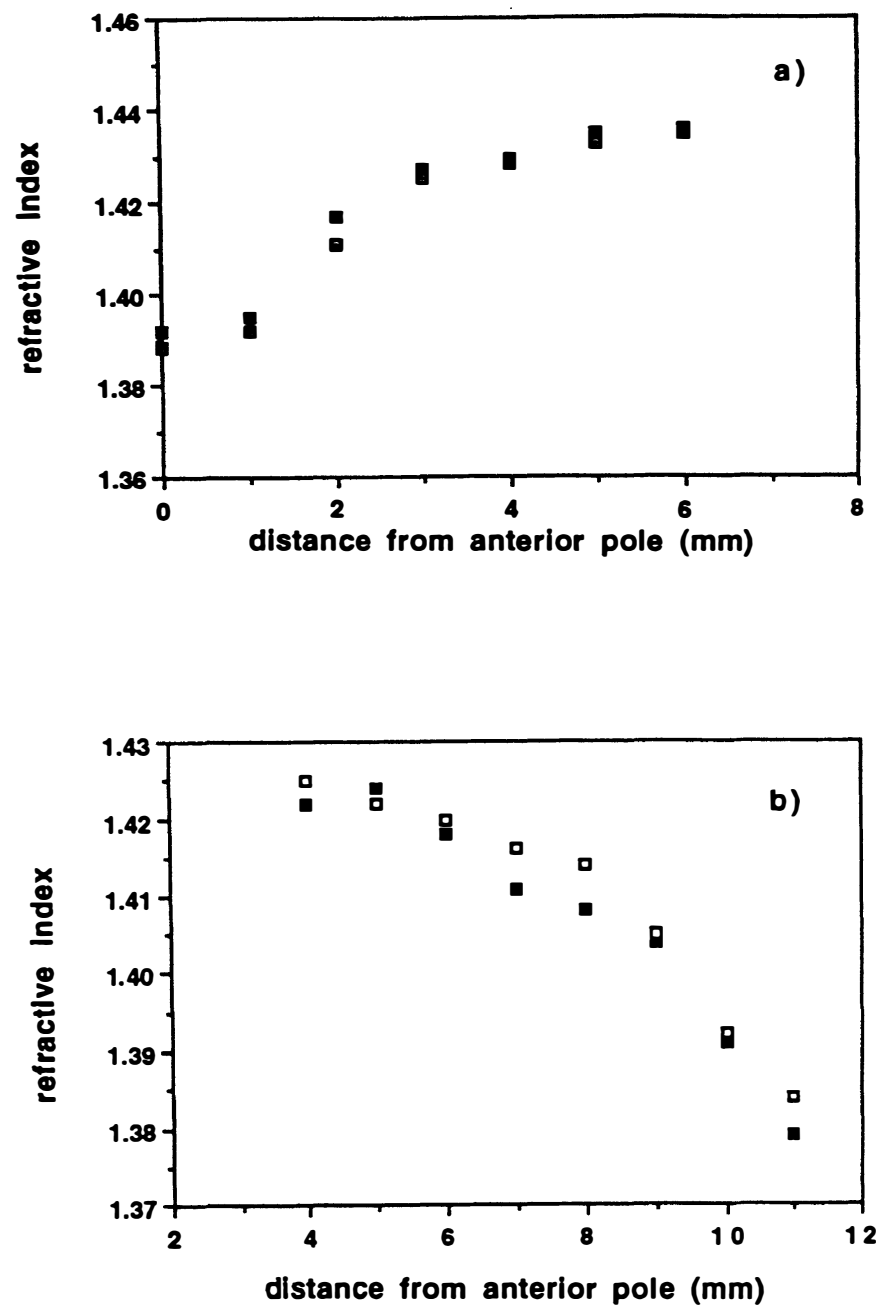

Fig. 2. Refractive index gradients in the (a) anterior sagittal sections of two bovine lenses weighing $2.086 \mathrm{~g}$ (filled squares) and $2.089 \mathrm{~g}$ (open squares), and (b) posterior sagittal sections of two bovine lenses weighing $1.818 \mathrm{~g}$ (filled squares) and $1.850 \mathrm{~g}$ (open squares). Gradients are plotted against distance from the anterior pole.

the anterior sections are almost superimposable (Fig. $1 \mathrm{a})$, while there is a small discrepancy between the values from the two data sets at $1-2 \mathrm{~mm}$ from the posterior pole (Fig. 1b). Both sets of gradients peak between 4.5 and $5.5 \mathrm{~mm}$ from the anterior pole at an index value of about 1.435 .

There is evidence of some variations in index gradient even between lenses of very similar weights. Fig. 2a shows anterior gradients from two lenses of weight $2.086 \mathrm{~g}$ and $2.089 \mathrm{~g}$. Here there is very little difference between the two gradients. However, in Fig. 2b, which shows index variations in the posterior lens sections (plotted against distance from the anterior pole) from two lenses of weight $1.818 \mathrm{~g}$ and $1.850 \mathrm{~g}$, the refractive index magnitudes are discrepant between $6 \mathrm{~mm}$ and $9 \mathrm{~mm}$ from the anterior pole.

Combined results for all the lenses are shown in Fig. 3. Different symbols have been used to distinguish anterior and posterior gradients. The 


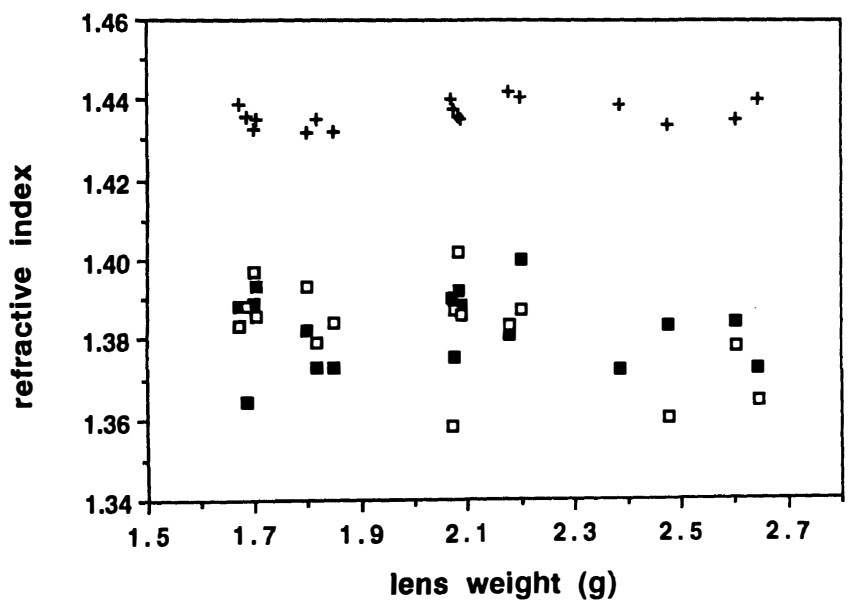

Fig. 4. Refractive index values at the centre of the equatorial plane (crosses), the anterior pole (filled squares) and at the posterior poles (open squares) for all lenses studied, plotted against lens weight.

data are scattered about curves which can be fitted to the following second-order polynomials:

anterior

$$
\begin{gathered}
y=1.3790+0.018494 x-0.0014925 x^{2} \\
R^{2}=0.897 p<0.005
\end{gathered}
$$

posterior

$$
\begin{gathered}
y=1.4102+0.009647 x-0.0010741 x^{2} \\
R^{2}=0.831 p<<0.005
\end{gathered}
$$

The peak index values are clustered around 1.435 but there is a much greater spread of values at both poles. This is illustrated in Fig. 4, which shows the peak and anterior and posterior edge index magnitudes. The apparent scatter in central index values seen in Fig. 3 is due to differences in lens sizes and hence variations in the position of the peak index value along the abscissa. However, this peak lies between 4.5 and $5.5 \mathrm{~mm}$ from the anterior edge, which is consistent with values for anterior sagittal radii, previously measured, and corresponds to the approximate position of the equatorial plane. ${ }^{17}$ While there may be individual variations which would contribute to scatter in the data, it is unlikely that this alone is the reason for the wide range of edge index values. The spread may be related in part to the degree of hydration of the surface cells and possible changes in the hydration when the capsule is ruptured. This is a factor which can only be eliminated by conducting the experiment in an environment of controlled and constant hydration. Another possible factor is local disruption to the surface layers of lens tissue caused by the piercing of the lens capsule.

Table I shows the equations, correlation coefficients and statistical significance of linear functions fitted to all data sets in Fig. 4. Only the posterior data set from this study shows any statistically significant variation in index with lens weight (if $p=0.05$ is taken as the cutoff for significance).
Table I. Equations and statistical analysis pertaining to Fig. 4

\begin{tabular}{lccc}
\hline Location & $y=a+b x$ & $R^{2}$ & $p$ \\
\hline Centre & $y=1.4295+0.00344 x$ & 0.126 & $<0.1$ \\
Anterior & $y=1.3857-0.00164 x$ & 0.003 & $<0.45$ \\
Posterior & $y=1.4247-0.02083 x$ & 0.298 & $<0.05$ \\
\hline
\end{tabular}

$y$ represents the refractive index; $x$ represents lens weight.

When the refractive index values measured at the anterior and posterior poles in this study (the surface index values) are compared with data previously obtained on the surfaces of decapsulated bovine lenses $^{16}$ (Fig. 5a, anterior pole; Fig. 5b, posterior pole), it is clear that the latter sets are higher in value and less scattered. This may be related to the fact that the lenses measured previously were decapsulated and the exposed lens surfaces became slightly dehydrated. However, the decapsulated lenses were from a lower weight range (1.590-1.966 g) than those used in this study (1.670-2.646 g) and the discrepancies between the two sets of data may be revealing a gross difference in surface index values with size and hence with age, since the size of the bovine lens has
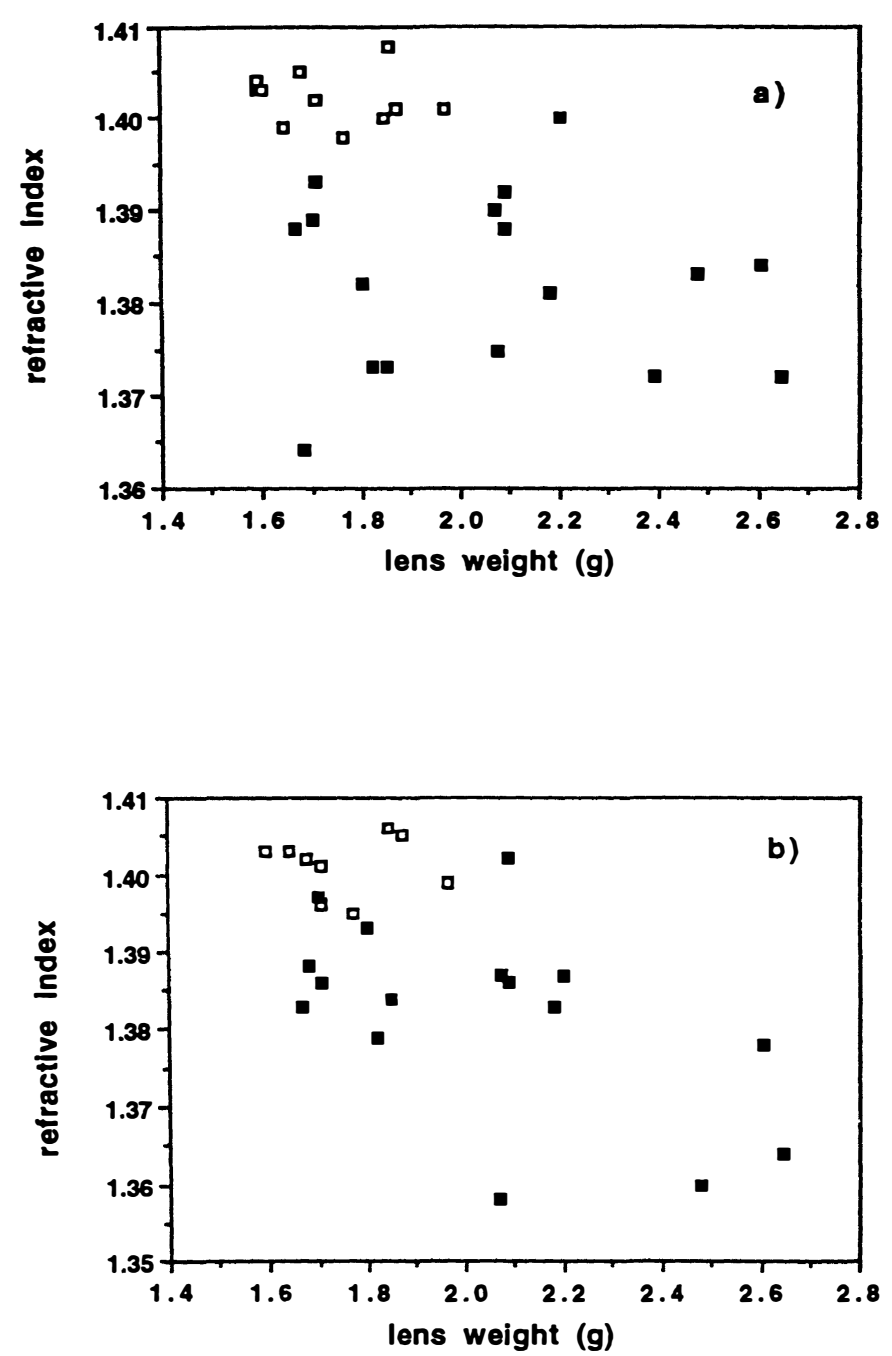

Fig. 5. Refractive index at the (a) anterior and (b) posterior poles plotted against lens weight. Results from this study (filled squares) are compared with results from a previous study (Pierscionek, ${ }^{16}$ open squares). 


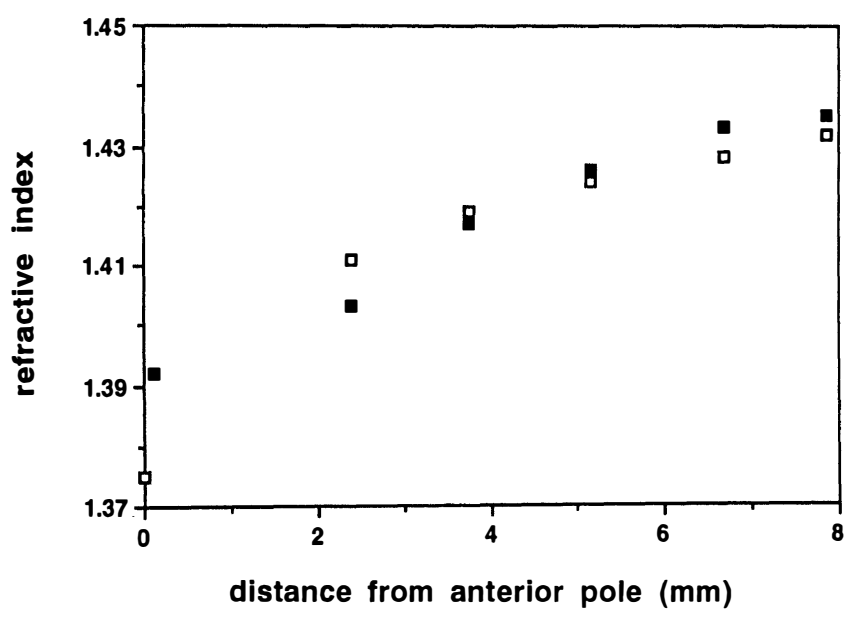

Fig. 6. Consecutive measurements of refractive index in the anterior section of a $1.76 \mathrm{~g}$ bovine lens. The filled squares represent the second set of measurements.

been shown to be related to lens age. ${ }^{18}$

In order to assess the extent of tissue disruption within the lens and hence any changes in refractive index after passage of the probe, two sets of readings were taken in the anterior section of a $1.76 \mathrm{~g}$ lens: after the first set of measurements the probe was removed and then readings were repeated at the same points. The results are shown in Fig. 6. Apart from the discrepancy between the refractive index at the outermost point, where dehydration would have the greatest effect, most of the other values are superimposable.

\section{DISCUSSION}

The results of this study provide the first accurately localised measurements of refractive index along the optic axis of the bovine lens.

Before experimentation commenced, the major reservation concerning this method of fibre optic sensing was that there may be some adhesion of outer layer tissue to the end of the probe and that this could be carried through the layers of the lens. If this had occurred, the results would show no variation in refractive index across the lens since the recorded reflectance value comes from the medium which is in contact with the fibre. Similarly, significant mixing of cytoplasm from adjacent cells or tissue distortion would not have produced index gradients of a similar shape (polynomials of second order) to those previously determined with ray trace analysis ${ }^{11,19}$ but rather skewed gradients resulting from smearing of cellular contents. Indeed the results show consistent variations in index along the optic axis between lenses and even within a single lens. The needle probe does not compress the tissue but rather 'cuts' through it. There is no resistance to the movement of the probe through the lens and since it is suspended by attachment to the micrometer, it rests on the tissue layer at which a measurement is to be taken, without pressing it. The probing action can only cause an alteration of local tissue order but, as is evident in Fig. 6, passage of the probe through a lens did not alter subsequent refractive index measurements, suggesting that there were no major structural disturbances to the tissue.

Over the range of lenses used in this study, there do not appear to be any obvious trends in the gradient shape or magnitude which may be related to lens weight and hence lens age. The spread of data points seen when gradients from all lenses are combined (Fig. 3), and indeed differences in gradients between lenses of very similar weights (Fig. 2a and b), indicate that, in addition to system errors, there may be individual variations which mask any age-related trends. Such variations between lenses are not surprising given that for any organ or biological tissue, values of parameters are expected to deviate within a given range. There is an asymmetry between the anterior and posterior refractive index gradients which, given the asymmetry in lens shape, is not surprising: the highest index values should be close to the equatorial plane which contains at its centre the oldest, densest tissue in the lens. This is indeed supported by the measurements.

The range of highest index values is between 1.432 in $1.850 \mathrm{~g}$ and $1.801 \mathrm{~g}$ lenses and 1.442 in a $2.18 \mathrm{~g}$ lens. This compares well with the results of Rink ${ }^{20}$ for measurements of water gradients in bovine lenses. He found that in the centre, older lenses ( $2.4 \mathrm{~g}$ and greater in weight) contained $52 \%$ water, young lenses (less than $1.8 \mathrm{~g}$ in weight) contained $57 \%$ water and for lenses between these weights the value was $54 \%$. Refractive indices calculated from these values (using $0.72 \mathrm{ml} / \mathrm{g}$ as the partial specific volume of proteins, 1.333 as the refractive index of water and $0.1901 \mathrm{ml} / \mathrm{g}$ as the specific refractive increments for crystallins at the centre of the lens, taking into account the individual increments ${ }^{21}$ and the proportions of the different protein classe ${ }^{22}$ ) are $1.438,1.433$ and 1.426 for $52 \%, 54 \%$ and $57 \%$ water respectively. Rink's study covered a greater age range (0.3-22 years) and hence was able to discern general trends from among individual variability.

The findings of Sivak and Mandelman's study, ${ }^{23}$ using Pulfrich refractometry on bisected lenses, show a much higher magnitude of refractive index (1.4593 for a wavelength of $650 \mathrm{~nm}$ ) in the innermost region of the bovine lens. The value is a mean of averaged values from two lenses (each average was from three readings) but weights and ages of the samples are not given. Although there is a very large standard deviation in the results of Sivak and Mandelman, this cannot explain the discrepancy between their value and the values of Rink and those of this study. 
Perhaps the most pertinent point to remember is that refractive index is dependent on a number of factors and will alter with conditions. It is possible that bisecting the lens caused dehydration of the exposed surface and resulted in the measurement of higher refractive index. The reported value of Sivak and Mandelman's is therefore appropriate for a bisected lens.

Castoro and Bettelheim, ${ }^{24}$ who measured water gradients in adult bovine lenses, reported about 74$79 \%$ water content at the lens surfaces. This would give refractive index values of 1.385-1.374 using 1.331 for the refractive index of water, as given by Castoro and Bettelheim for the value of free water at $37^{\circ} \mathrm{C}$. These values are within the range of values reported in this work, albeit at the lower end.

In comparison with the other techniques mentioned, the major advantage offered by the optic fibre reflectometer is accuracy in localisation of the sampled points. In addition, this method of optical fibre sensing involves a great deal less violation of the tissue than slicing or bisection and hence results reflect more closely the in vivo situation. However, while the optic fibre sensor allows a direct and highly localised and sensitive measurement of refractive index to be made, the degree of sensitivity may contribute to the scatter in the data as seen in the edge values. Differences in hydration, even concentrated over a very small region, will result in variations in refractive index and this may explain the higher index values measured at the anterior and posterior polar surfaces of decapsulated bovine lenses $^{16}$ when compared with the surface index values found in this work.

Early work on the refractive index of the bovine lens, which adopted a totally non-destructive approach and determined refractive index from ray trace parameters, ${ }^{11,19}$ had to rely on certain assumptions in the mathematical analysis. One of these was the assumption of contours of equal refractive index within the lens, which followed the surface shape (isoindicial contours) ${ }^{10,11,13}$ This assumption seemed reasonable in light of the growth mode of the lens and it simplified the approach: because of the shape asymmetry in the sagittal plane, measurements were taken in the equatorial plane, in which the lens shape is symmetrical, and assuming isoindicial contours, results were transposed to the sagittal plane. ${ }^{11}$ However, recent work has shown that the assumption of isoindicial contours may not be valid in all regions of the lens (Pierscionek and D. Y. C. Chan, unpublished) and hence while the ray tracing may be applicable to the equatorial plane, transposition to the sagittal plane needs to be checked with another, direct method. The optic fibre sensor provides a very suitable way of measuring refractive index of the lens at any chosen point within the tissue and this may be the ideal method for determining the form of the isoindicial contours.

\section{APPENDIX}

The final signal detected by the power meter $D$ is reduced by a number of factors. It has been expressed as: ${ }^{25}$

$$
D=I(R+S)
$$

where $I$ is the product of the input power (watts), light losses and attenuations in the forward direction of travel, $R$ is the Fresnel reflectance from the end of the experimental arm, and $S$ is the sum of the spurious contributions from backscattered and ambient light. The refractive index of a sample is determined by the following equation: ${ }^{25}$

$$
n=n_{\mathrm{e}} \frac{\left.\left\{1-\left[S^{\prime}+g\right) K-S^{\prime}\right]^{1 / 2}\right\}}{\left\{1+\left[\left(S^{\prime}+g\right) K-S^{\prime}\right]^{1 / 2}\right\}}
$$

where $n_{\mathrm{e}}$ is the effective refractive index of the fibre core, $S^{\prime}$ is the sum of the attenuated spurious components, $g$ is equal to $\left[\left(n_{\mathrm{e}}-n_{\mathrm{a}}\right) /\left(n_{\mathrm{e}}+n_{\mathrm{a}}\right)\right]^{2}\left(n_{\mathrm{a}}\right.$ is the refractive index of air), and $K$ is the ratio of power measured in air to that in the sample.

When the refractive index of the sample is closely matched to the effective refractive index of the fibre core, the reflection at the interface of the fibre end and the sample is very low and the power detected is largely from the spurious (noise) components. The ratio of power detected in the matching liquid to that in air is used to calibrate the noise level, $S^{\prime}$. The liquid used is $\mathrm{CCl}_{4}$, which has a refractive index of 1.4603 at $20{ }^{\circ} \mathrm{C}$ for $589 \mathrm{~nm}$, as measured with the Abbe refractometer. The effective refractive index of the fibre is $1.4625(633 \mathrm{~nm})$ as provided by the distributor (Optical Waveguides Australia, Noble Park, Victoria, Australia). (Readings were in $\mathrm{dBm}$ and had to be converted to milliwatts for calculations $\left(\mathrm{dBm}=10 \log _{10} P\right.$, where $P$ is the power in milliwatts.)

The author is supported by the National Health and Medical Research Council (R. D. Wright fellowship).

Key words: Bovine, Fibre optic sensor, Lens, Optic axis, Refractive index.

\section{REFERENCES}

1. Huggert A. On the form of the iso-indicial surfaces of the human crystalline lens. Acta Ophthalmol Suppl 1948;30:1-126.

2. Nakao S, Fujimoto S, Nagata R, Iwata K. Model of refractive index distribution in the rabbit crystalline lens. J Opt Soc Am 1968;58:1125-30.

3. Nakao $S$, Ono $T$, Nagata $R$, Iwata $K$. Model of refractive indices in the human crystalline lens. Jpn J Clin Ophthalmol 1969;23:903-6.

4. Philipson B. Distribution of protein within the normal rat lens. Invest Ophthalmol 1969;8:258-70.

5. Bando M, Nakajima A, Nakagawa M, Hiroaka T. 
Measurement of protein distribution in human lens by microspectrophotometry. Exp Eye Res 1976;22:389-92.

6. Palmer DA, Sivak J. Crystalline lens dispersion. J Opt Soc Am 1981;71:780-2.

7. Jagger WS. The refractive structure and optical properties of the isolated crystalline lens of the cat. Vision Res 1990;30:723-38.

8. Pomerantzeff O, Pankratov M, Wang G-J, Dufault P. Wide-angle optical model of the eye. Am J Optom Physiol Opt 1984;61:166-76.

9. Campbell MCW, Hughes A. An analytic gradient index schematic lens and eye for the rat which predicts aberrations for finite pupils. Vision Res 1981;21:1129-48.

10. Campbell MCW. Measurement of refractive index in an intact crystalline lens. Vision Res 1984;24:409-15.

11. Pierscionek BK, Chan DYC, Ennis JP, Smith G, Augusteyn RC. A non-destructive method of constructing three-dimensional gradient index models for crystalline lenses. I. Theory and experiment. Am J Optom Physiol Opt 1988;65:481-91.

12. Axelrod D, Lerner D, Sands PJ. Refractive index within the lens of a goldfish eye determined from the paths of thin laser beams. Vision Res 1988;28:57-65.

13. Pierscionek BK, Chan DYC. Refractive index gradient of human lenses. Optom Vision Sci 1989;66:822-9.

14. Pierscionek BK. Surface refractive index of the eye lens determined with an optic fibre sensor. J Opt Soc Am A 1993;10:1867-71.

15. Pierscionek BK. Refractive index of the human lens surface measured with an optic fibre sensor. Ophthalmic Res 1994;26:32-6.

16. Pierscionek BK. Refractive index of decapsulated bovine lens surfaces measured with a reflectometric sensor. Vision Res 1994;34:1927-33.

17. Pierscionek BK, Augusteyn RC. Growth related changes to functional parameters in the bovine lens. Biochim Biophys Acta 1992;1116:283-90.

18. Hockwin O. Age changes of lens metabolism. Altern Entwickl 1971;1:95-129.

19. Pierscionek BK. Growth and ageing effects on the refractive index gradient in the equatorial plane of the bovine lens. Vision Res 1989;29:1759-66.

20. Rink $H$. The water content in bovine lenses during aging. Interdiscipl Topics Gerontol 1978;12:271-7.

21. Pierscionek B, Smith G, Augusteyn RC. The refractive increments of bovine $\alpha$ - , $\beta$ - and $\gamma$-crystallins. Vision Res 1987;27:1539-41.

22. Pierscionek B, Augusteyn RC. Protein distribution patterns in concentric layers from single bovine lenses: changes with development and ageing. Curr Eye Res 1988;7:11-23.

23. Sivak J, Mandelman T. Chromatic dispersion of the ocular media. Vision Res 1982;22:997-1003.

24. Castoro JA, Bettelheim FA. Water gradients across bovine lenses. Exp Eye Res 1987;45:191-5.

25. Smith GE, White IA. Optical fibre reflectometer sensor. Presented at 17th Australian Conference on Optical Fibre Technology, 1992:198-201. 